\title{
Electrical spin injection into InGaAs/GaAs quantum wells: a comparison between MgO tunnel barriers grown by sputtering and molecular beam epitaxy methods
}

P. Barate ${ }^{1}$, S. Liang 2 , T. T. Zhang ${ }^{1}$, J. Frougier ${ }^{3}$, M. Vidal ${ }^{1}$, P. Renucci ${ }^{1}$, X. Devaux ${ }^{2}$, B. Xu X $^{4}$, H. Jaffrès ${ }^{3}$, J. M.


${ }^{1}$ Université de Toulouse, INSA-CNRS-UPS, LPCNO, 135 avenue de Rangueil, 31077 Toulouse, France ${ }^{2}$ Institut Jean Lamour, UMR 7198, CNRS-Nancy Université, BP 239, 54506 Vandoeuvre, France

${ }^{3}$ Unité Mixte de Physique CNRS/Thales and Université Paris-Sud 11, 1 avenue A. Fresnel, 91767 Palaiseau, France

${ }^{4}$ Key Laboratory of Semiconductor Materials Science, Institute of Semiconductors, Chinese Academy of Sciences, P. O. Box 912, Beijing 100083, China

${ }^{5}$ Institut des NanoSciences de Paris, UPMC, CNRS UMR 7588, 4 place Jussieu, 75005 Paris, France

${ }^{6}$ Beijing National Laboratory for Condensed Matter Physics, Institute of Physics, Chinese Academy of Sciences, P.O. Box 603, Beijing 100190, China

*Corresponding author: yuan.lu@univ-lorraine.fr

\begin{abstract}
An efficient electrical spin injection into an $\mathrm{InGaAs} / \mathrm{GaAs}$ quantum well light emitting diode is demonstrated thanks to a $\mathrm{CoFeB} / \mathrm{MgO}$ spin injector. The textured $\mathrm{MgO}$ tunnel barrier is fabricated by two different techniques: sputtering and molecular beam epitaxy (MBE). The maximal spin injection efficiency is comparable for both methods. Additionally, the effect of annealing is also investigated for the two types of samples. Both samples show the same trend: an increase of the electroluminescence circular polarization $\left(P_{\mathrm{c}}\right)$ with the increase of annealing temperature, followed by a saturation of $P_{\mathrm{c}}$ beyond $350^{\circ} \mathrm{C}$ annealing. Since the increase of $P_{\mathrm{c}}$ starts well below the crystallization temperature of the full $\mathrm{CoFeB}$ bulk layer, this trend could be mainly due to an improvement of chemical structure at the top $\mathrm{CoFeB} / \mathrm{MgO}$ interface. This study reveals that the control of $\mathrm{CoFeB} / \mathrm{MgO}$ interface is essential important for an optimal spin injection into semiconductor.
\end{abstract}


Efficient injection of spin-polarized electrons from a ferromagnetic (FM) source into a semiconducting heterostructure is a prerequisite for the realization of a large number of new spintronic devices including spintransistors $^{1}$, spin light emitting diodes (spin-LEDs) ${ }^{2-12}$ and spin-lasers ${ }^{13}$. It has been shown that inserting a thin MgO layer between the FM contact and semiconductor can circumvent the problem of conductivity mismatch between FM metals and semiconductors, resulting in a very high spin injection efficiency ${ }^{14}$. In particular, the $\mathrm{CoFe} / \mathrm{MgO}$ injector has exhibited the highest spin injection yield at room temperature up to now (the electroluminescence polarization can reach $32 \%$ at $300 \mathrm{~K})^{6}$. This magnetic tunnel injector is constructed with two interfaces: the ferromagnetic metal (FM)/MgO and the $\mathrm{MgO} /$ semiconductor interfaces. It is still an open question to determine the role of interface for an optimal spin injection. Moreover, although the symmetry rules for the tunneling transfer of carriers in magnetic tunnel junctions (MTJs) with an MgO tunneling barrier ${ }^{15-17}$ are well known for standard MTJ systems, it is significantly less understood if the $\mathrm{MgO}$ can act as a relevant spin filter to enhance the spin injection efficiency into semiconductor. In this letter, we propose to tackle these points by studying spin-LED systems with $\mathrm{CoFeB} / \mathrm{MgO} / \mathrm{GaAs}$ injectors, where the $\mathrm{MgO}$ barrier is grown by two different techniques: sputtering and molecular beam epitaxy (MBE). This results in the different quality of $\mathrm{MgO} / \mathrm{GaAs}$ and $\mathrm{CoFeB} / \mathrm{MgO}$ interface prepared by the two different techniques, which can allow us to examine which interface is crucial for spin injection. In addition, $\mathrm{CoFeB}$ is a promising candidate as a ferromagnetic injector, because after $350^{\circ} \mathrm{C}$ annealing $\mathrm{CoFeB}$ is easy to form a bcc grain-to-grain epitaxial crystalline phase on $\mathrm{MgO} 18$. This improvement by annealing has been particularly observed in MTJs, where $600 \%$ tunnel magnetoresistance (TMR) was measured on $\mathrm{CoFeB} / \mathrm{MgO} / \mathrm{CoFeB}$ junctions ${ }^{19}$ instead of $180 \%$ observed on $\mathrm{CoFe} / \mathrm{MgO} / \mathrm{CoFe}$ junctions ${ }^{17}$. Studying the annealing effect on these spin-LED with $\mathrm{CoFeB} / \mathrm{MgO}$ injectors by correlating the circular light polarization with the crystallization state of $\mathrm{CoFeB}^{20,21}$ will allow us to examine the key role of interface and further determine if the $\mathrm{MgO}$ spin filtering effect exists for spininjection into semiconductors.

The quantum well (QW) $p$-i-n LED device has the following structure sequence (Fig. 1): $p$-GaAs:Zn (001) substrate $\left(p=2 \times 10^{19} \mathrm{~cm}^{-3}\right) / 500 \mathrm{~nm} p$-GaAs:Be $\left(p=2 \times 10^{19} \mathrm{~cm}^{-3}\right) / 200 \mathrm{~nm} p$-GaAs:Be $\left(p=2 \times 10^{18} \mathrm{~cm}^{-3}\right) / 50 \mathrm{~nm}$ undoped GaAs $/ 10 \mathrm{~nm}$ undoped $\operatorname{In}_{0.1} \mathrm{Ga}_{0.9} \mathrm{As} / 50 \mathrm{~nm}$ undoped GaAs $/ 50 \mathrm{~nm} n$-GaAs:Si $\left(n=1 \times 10^{16} \mathrm{~cm}^{-3}\right)$. The LED surface was passivated with arsenic in the III-V MBE chamber and then transferred through air into another MBE-sputtering interconnected system. The arsenic capping layer was firstly desorbed at $300^{\circ} \mathrm{C}$ in the MBE chamber. Two methods were then used to grow the $\mathrm{MgO}$ tunneling barrier layer. Either $\mathrm{MgO}$ is grown at $250^{\circ} \mathrm{C}$ in the $\mathrm{MBE}$ chamber after arsenic desorption, or the sample is transferred into the sputtering chamber through vacuum to grow the MgO layer. In both cases, $\mathrm{MgO}$ layer has identical thickness of $2.5 \mathrm{~nm}$. Finally, the $3 \mathrm{~nm}$ CoFeB ferromagnetic contact and $5 \mathrm{~nm}$ 
Ta protection layer were deposited by sputtering. Hereafter, we will name the samples "MBE" and "sputtering" to refer to two different spin-LEDs with $\mathrm{MgO}$ prepared by MBE and sputtering techniques, respectively. These two growth techniques produce a different quality of $\mathrm{MgO}$ barrier and also a different quality of interface (see in Fig. 1 "top and bottom interface"). It will allow us to determine which factor is crucial for the efficient electrical spin injection, knowing that the thicknesses of all layers involved in the injector are the same for both types of sample. $300 \mu \mathrm{m}$ diameter circular mesas were then processed using standard UV photolithography and etching techniques. In the end, the processed wafers were cut into small pieces to perform rapid temperature annealing (RTA) at different temperatures $\left(T_{\mathrm{a}}\right)$ for one minute. The RTA procedure is a good way to promote the crystallization of $\mathrm{CoFeB}^{22}$ while preventing a change to the LED optical characteristics.

The electroluminescence (EL) results are a first test of the difference between the two barrier growth methods. A more detailed work condition of the polarization resolved EL measurements can be found in a previous work ${ }^{8}$ by our group. Insets of Fig. 2(a) show a typical CW EL spectra from a spin-LED with a sputtered MgO tunnel barrier $\left(T_{\mathrm{a}}=350^{\circ} \mathrm{C}\right)$ acquired at $25 \mathrm{~K}$ under a bias of $V_{\text {bias }}=2.4 \mathrm{~V}$ for $B=0 \mathrm{~T}$ (top inset) and $B=0.8 \mathrm{~T}$ (bottom inset). The EL circular polarization $P_{\mathrm{c}}$ is defined as $P_{\mathrm{c}}=\left(I^{\sigma^{+}}-I^{\sigma-}\right) /\left(I^{\sigma+}+I^{\sigma-}\right)$ where $I^{\sigma^{+}}$and $I^{\sigma-}$ are the intensities of the right and left circularly polarized components of the luminescence, respectively. Whereas the heavy-hole exciton (XH) EL peak observed at $878 \mathrm{~nm}$ does not show any circular polarization at zero magnetic field (top inset), the $P_{\mathrm{c}}$ reaches $24 \% \pm$ $1 \%$ under $B=0.8 \mathrm{~T}$ (bottom inset). Additionally, the magnetic circular dichroism is less than $1 \%$ at $0.8 \mathrm{~T}$ thanks to a measurement based on a linearly polarized He-Ne $\operatorname{laser}^{23}$. The measured $P_{\mathrm{c}}$ increases with the applied longitudinal magnetic field [Fig. 2(a)], due to the progressive increase of the projection of the out-of-plane magnetization (the magnetization of $\mathrm{CoFeB}$ layer is within the plane at zero magnetic field), which can be explained by the optical selection rules applied to the $\mathrm{QW}^{24}$. In Fig. 2(b), we present a systematic study of the influence of the post-annealing temperature on $P_{\mathrm{c}}$ for the two kinds of spin-LED samples. For sputtered tunnel barriers, we observe a clear improvement of the measured EL circular polarization rate from $13.5 \pm 1 \%$ before annealing up to $24 \pm 1 \%$ for the optimal $T_{\mathrm{a}}=350^{\circ} \mathrm{C}$, following by a decrease of $P_{\mathrm{c}}$ with $T_{\mathrm{a}}=380^{\circ} \mathrm{C}$. This trend is similar for the sample based on a MBE grown barrier, with a slightly lower optimal polarization of $20 \pm 1 \%$ [the corresponding spectra are shown in the inset of Fig. 2(b)]. The behavior with annealing is similar to the one observed by Wang et al. ${ }^{25}$ for spin-LED with $\mathrm{CoFe} / \mathrm{MgO}$ injectors, but the variation in our spin-LED is much remarkable.

The use of spin-LEDs as an optical means to quantify the electrical spin injection in GaAs is based on a straightforward relationship between the electron spin polarization $P_{\mathrm{e}}$ injected in the QW and the measured EL 
circular polarization $P_{\mathrm{c}}: P_{\mathrm{c}}=P_{\mathrm{e}} * F^{26,27}$. The $F$ factor takes into account the electron spin relaxation in the QW during the electron lifetime: $F=1 /\left(1+\tau / \tau_{\mathrm{s}}\right)$ with $\tau_{\mathrm{s}}$ spin relaxation time and $\tau$ electron lifetime in the $\mathrm{QW}^{26,27}$. It is important to determine if the observed large improvement of $P_{\mathrm{c}}$ as a function of annealing temperature displayed in Fig. 2(b) is due to a real improvement of $P_{\mathrm{e}}$, or only due to the impact of the annealing process on the QW properties. Therefore, a systematic measurement of the $F$ factor as a function of the annealing temperature was performed by measuring $\tau_{\mathrm{s}}$ and $\tau$ in a bare $p-i-n$ LED sample by time and polarization resolved photoluminescence (TRPL). Detailed experimental conditions can be found elsewhere ${ }^{8}$. As shown in Fig. 2(c) as an example for the bare $p-i-n$ LED with $T_{\mathrm{a}}=350^{\circ} \mathrm{C}$, the electron lifetime $\tau$ can be determined from the decay of the sum of $I^{\sigma+}$ and $I^{\sigma-}$ to be $180 \pm 10$ ps at $1 / \mathrm{e}$, and the spin relaxation time $\tau_{\mathrm{s}}$ can be determined from the decay of the circular polarization to be $425 \pm 50$ ps at $1 /$ e. So the $F$ factor can be determined to be 0.7 . The measured $\tau$ and $\tau_{\mathrm{s}}$ and the deduced $F$ factor as a function of $T_{\mathrm{a}}$ are summarized in the inset of Fig. 2(d). For better comparison of the variation of $P_{\mathrm{c}}$ and $F$ factor as a function of $T_{\mathrm{a}}$, the relative change compared to the case before annealing (BA) is plotted in Fig. 2(d). It clearly shows that the relative improvement of the circular polarization $\left[P_{\mathrm{c}}\left(T_{\mathrm{a}}\right)-P_{\mathrm{c}}(\mathrm{BA})\right] / P_{\mathrm{c}}(\mathrm{BA})$ reaches about $80 \%$ between $P_{\mathrm{c}}(\mathrm{BA})$ and $P_{\mathrm{c}}\left(T_{\mathrm{a}}=350^{\circ} \mathrm{C}\right)$ for sputtered samples. However, the relative variation of the $F$ factor $\left[F\left(T_{\mathrm{a}}\right)-F(\mathrm{BA})\right] / F(\mathrm{BA})$ is much weaker [less than $10 \%$ between $F(\mathrm{BA})$ and $F\left(T_{\mathrm{a}}=350^{\circ} \mathrm{C}\right)$ ] and is even negative. So the large improvement of $P_{\mathrm{c}}$ observed in Fig. 2(b) is due to a real improvement from the spin injector part $\left(P_{\mathrm{e}}\right)$ because of the small (and opposite) variation of the $F$ factor.

In the EL measurement, the maximum out-of-plane field is limited at $0.8 \mathrm{~T}$, which cannot fully saturate the CoFeB magnetization. To eliminate the possibility of the increase of $P_{\mathrm{c}}$ due to the change of the saturation field after annealing, a superconducting quantum interference device magnetometer (SQUID) was employed to measure the magnetization of film sample of a sputtered spin-LED in out-of-plane configuration. As shown in Fig. 3(a), the magnetization is quasi linear between $-1.3 \mathrm{~T}$ and $1.3 \mathrm{~T}$ as a function of magnetic field for before annealing and $T_{\mathrm{a}}=275^{\circ} \mathrm{C}$ and becomes non-linear with $T_{\mathrm{a}}=350^{\circ} \mathrm{C}$. Let's note that the saturation field measured is $\sim 1.3 \mathrm{~T}$ for $T_{\mathrm{a}}$ below $300^{\circ} \mathrm{C}$ and $\sim 1.75 \mathrm{~T}$ for $350^{\circ} \mathrm{C}$. In order to extrapolate $P_{\mathrm{c}}$ at saturation, the $P_{\mathrm{c}}$ value at $0.8 \mathrm{~T}$ is multiplied by a ratio $M_{\text {sat }} / M(0.8 \mathrm{~T})$ based on the results obtained by SQUID. The extrapolation leads to a rough estimation of $P_{\mathrm{c}}$ at saturation of about $42.0 \%$ at $25 \mathrm{~K}$ for spin-LED with sputtered $\mathrm{MgO}$ after $350^{\circ} \mathrm{C}$ annealing, proving the high efficiency of $\mathrm{CoFeB} / \mathrm{MgO}$ injector. This result is close to the best obtained by Jiang et al. $\left(P_{\mathrm{c}}=50 \%\right.$ at low temperature with $\mathrm{CoFe} / \mathrm{MgO} / \mathrm{AlGaAs}$ injector ${ }^{6}$ ) and is larger than the one reported very recently by $\mathrm{Li}$ et al. ${ }^{28}$ with a Schottky barrier as spin injector $\left(P_{\mathrm{c}}=25 \%\right)$. As shown in Fig. 3(a) with dashed line, the ratio $M_{\text {sat }} M(0.8 \mathrm{~T})$ does 
not depend on the annealing temperature, which validates the direct comparison of $P_{\mathrm{c}}$ measured at $0.8 \mathrm{~T}$ as function of $T_{\mathrm{a}}$ in Fig. 2(b). The non-linear $M-H$ curve for $T_{\mathrm{a}}=350^{\circ} \mathrm{C}$ in fact reflects the crystallization of CoFeB layer ${ }^{20}$. To further clarify the influence of the annealing temperature on the crystallization of CoFeB layer, SQUID measurements were performed in an in-plane configuration to check the coercivity $H_{\mathrm{c}}$ of CoFeB layer. Fig. 3(c) displays the hysteresis cycles of two types of spin-LEDs annealed at different temperatures. Fig. 3(b) summarizes the evolution of $H_{\mathrm{c}}$ as a function of $T_{\mathrm{a}}$. When $T_{\mathrm{a}}$ is above $300^{\circ} \mathrm{C}, H_{\mathrm{c}}$ increases in both types of samples. This is a strong indication of the beginning of the $\mathrm{CoFeB}$ crystallization $^{22}$. It is noted that the MBE sample has a relative larger $H_{\mathrm{c}}$ than the sputtering sample after annealing, which could be related to the different magnetic domain structures in CoFeB layer (discussed below). The increase of the coercivity field with $T_{\mathrm{a}}$ saturates around $350^{\circ} \mathrm{C}$, signifying a full crystallization of CoFeB layer, which is in good agreement with reported crystallization temperature ${ }^{20}$. However, these EL measurements indicate that $P_{\mathrm{c}}$ increases begin far below $300^{\circ} \mathrm{C}$, with saturation at $350^{\circ} \mathrm{C}$ when the crystallization is finished. Therefore it is clear that the increase of $P_{\mathrm{c}}$ is not due to the crystallization of the whole $\mathrm{CoFeB}$ layer.

To better understand the effect of annealing on $P_{\mathrm{c}}$, high-resolved transmission electron microscopy (HRTEM) is used to examine the interfacial structural information. The good homogeneity of the structures is checked on the low magnification images in the insets of Fig. 4(d) [for the sputtering sample annealed at $350^{\circ} \mathrm{C}$, the $\mathrm{MBE}$ sample has identical feature (not shown)]. Note also that the $\mathrm{MgO}$ thicknesses are identical for the two types of samples validating the direct comparison of the measured circular polarization from the two kinds of spin-LEDs in Fig. 2(b). First, we compare the TEM images for MBE samples before annealing [Fig. 4(a)] and after annealing at $350^{\circ} \mathrm{C}$ [Fig. 4(b)]. In both cases the $\mathrm{MgO} / \mathrm{GaAs}$ interface is sharp. The $\mathrm{CoFeB}$ layer is amorphous before annealing and fully crystallized after $350^{\circ} \mathrm{C}$ annealing, which further confirms the increase of $H_{\mathrm{c}}$ measured by SQUID is due to the crystallization of $\mathrm{CoFeB}$ layer. The selected zone FFT image shows a bcc structure of $\mathrm{CoFe}(\mathrm{B})$ on $\mathrm{MgO}$ [left inset of Fig. 4(b)]. The right inset of Fig. 4(b) shows the bright field HR-scanning TEM image, which allows us clearly identify the epitaxial crystalline orientation relationship between $\mathrm{GaAs}, \mathrm{MgO}$ and $\mathrm{CoFe}$ (B is absorbed by Ta): $\mathrm{GaAs}[100](100) / / \mathrm{MgO}[100](100) / / \mathrm{CoFe}[110](010)$. As $P_{\mathrm{c}}$ clearly increases with $T_{\mathrm{a}}$ for the MBE sample [Fig. 2(b)] whereas no observed change is found at the $\mathrm{MgO} / \mathrm{GaAs}$ interface and while a drastic change occurs at $\mathrm{CoFeB} / \mathrm{MgO}$ interface (evidenced by the $\mathrm{CoFeB}$ crystallization at $350^{\circ} \mathrm{C}$ ), one can infer that the $\mathrm{CoFeB} / \mathrm{MgO}$ interface is the crucial one for an optimal spin injection. A second observation reinforces this conclusion: in a comparison of the TEM images for the MBE [Fig. 4(a)] and sputtering [Fig. 4(c)] samples before annealing, a presence of a thin amorphous layer $(\sim 0.4 \mathrm{~nm})$ is detected at the $\mathrm{MgO} / \mathrm{GaAs}$ interface (bottom interface) for the 
sputtering sample [Fig. 4(c)]. The fact that $P_{\mathrm{c}}$ is comparable [Fig. 2(b)] before annealing for both samples (and even slightly better for a sputtered $\mathrm{MgO}$ barrier) whereas the quality of the $\mathrm{MgO} / \mathrm{GaAs}$ interface is much better by $\mathrm{MBE}$ also indicates that the influence of the $\mathrm{MgO} / \mathrm{GaAs}$ interface is weak and that the $\mathrm{CoFeB} / \mathrm{MgO}$ interface is the most important one for an efficient electrical spin injection. Finally, for the sputtering samples after $350^{\circ} \mathrm{C}$ annealing [Fig. 4(d)], the fully crystallization of CoFeB layer is also confirmed by the FFT image [left inset of Fig. 4(d)] as same as the MBE sample. It is also noted that the amorphous layer at $\mathrm{MgO} / \mathrm{GaAs}$ interface is much reduced after annealing. This amorphous layer could be due to the large kinetic energy of bombarded atoms during the sputtering growth process, which is then recrystallized after annealing.

To complete this study, reflection high energy electron diffraction (RHEED) measurements have been performed to check the $\mathrm{MgO}$ surface crystalline structure (top interface) prepared by the different methods. For MBE sample [insets of Fig. 4(a)], the RHEED patterns along GaAs [100] and [110] directions exhibit a monocrystalline spotty diffraction pattern, which further confirms the same in-plane epitaxial relationship $(\mathrm{GaAs}[100](100) / / \mathrm{MgO}[100](100))$ as that obtained from the HR-STEM image. However, for the sputtered MgO surface the RHEED images [insets of Fig. 4(c)] indicate a worse crystalline quality since some polycrystalline rings appear in the two directions. Nevertheless, the fact that the values of $P_{\mathrm{c}}$ are slightly larger for sputtering samples (whatever the annealing temperature is) despite this worse $\mathrm{MgO}$ quality indicates that the texture quality of the $\mathrm{MgO}$ barrier is less crucial for spin injection. As well known, during annealing, the Ta layer can absorb B atoms which results in the crystallization of $\mathrm{CoFeB}$ from the $\mathrm{MgO} / \mathrm{CoFeB}$ interface ${ }^{29}$. For the sputtering sample, the polycrystalline $\mathrm{MgO}$ grains observed before annealing will certainly induce high density of grains in the crystallized $\mathrm{CoFeB}$ layer after annealing due to the grain to grain epitaxial procedure ${ }^{18}$. The grain boundary related magnetic domain structures ${ }^{30}$ could explain the smaller coercivity observed in SQUID measurements [Fig. 3(b)] for the sputtering sample compared to the MBE sample.

To conclude, the quality of the $\mathrm{CoFeB} / \mathrm{MgO}$ interface seems to be crucial for an efficient electrical spin injection. As a large increase of the spin injection efficiency takes place below annealing at $300^{\circ} \mathrm{C}$, i.e. before crystallization of the whole $\mathrm{CoFeB}$ layer, we attribute this trend to an improvement of chemical bounds at the $\mathrm{CoFeB} / \mathrm{MgO}$ interface, as it was also observed for the TMR improvement in $\mathrm{MgO}$ MTJs below $300^{\circ} \mathrm{C}$ annealing ${ }^{31}$. The sub $300^{\circ} \mathrm{C}$ annealing can move the $\mathrm{Co}$ and $\mathrm{Fe}$ atoms toward to the $\mathrm{O}$ atom at $\mathrm{CoFeB} / \mathrm{MgO}$ interface, which efficiently enhances the interfacial spin polarization and interfacial perpendicular magnetization anisotropy ${ }^{32}$. Another point: whatever the annealing temperature is, the values of $P_{\mathrm{c}}$ are slightly larger for sputtering samples 
which undergo no interruption during the growth of the $\mathrm{CoFeB} / \mathrm{MgO}$ interface (while the MBE samples require a pause for sample transfer). This is another proof of the high importance of this interface. From $300^{\circ} \mathrm{C}$ to $350^{\circ} \mathrm{C}$ annealing, since the improvement of $P_{\mathrm{c}}$ is marginal, the $\mathrm{MgO}$ spin filter effect which can select the symmetry $\Delta_{1}$ electrons in CoFe band structure (possessing highest spin-polarization for tunneling) does not play an important role in our case. This could be related to the Ta diffusion toward $\mathrm{CoFeB} / \mathrm{MgO}$ interface ${ }^{19,33}$, which happens after high temperature annealing as indicating from the decrease of $P_{\mathrm{c}}$ with $T_{\mathrm{a}}=380^{\circ} \mathrm{C}$ [Fig. 2(b)]. Therefore, future enhancement of the $\mathrm{MgO}$ spin filtering effect, may rely upon the suppression the Ta diffusion (eg. replace with other metals for B absorption).

In summary, an efficient electrical spin injection from $\mathrm{CoFeB}$ into InGaAs/GaAs QW through $\mathrm{MgO}$ tunnel barriers is demonstrated $\left(P_{\mathrm{c}} \sim 24 \pm 1 \%\right.$ at $0.8 \mathrm{~T}$ corresponding to $P_{\mathrm{c}} \sim 42 \%$ at saturation at $\left.25 \mathrm{~K}\right)$. Spin-LEDs with MgO tunnel barriers are fabricated by two different techniques (sputtering and MBE). A systematic study of the impact of the post-annealing temperature on the two types of samples shows a similar increase of $P_{\mathrm{c}}$ with the increase of the annealing temperature and a comparable optimized spin injection efficiency for both methods in the range of $300-350^{\circ} \mathrm{C}$. This behavior is mainly attributed to the improvement of chemical structure at the top $\mathrm{CoFeB} / \mathrm{MgO}$ interface because the increase of $P_{\mathrm{c}}$ starts far below the crystallization temperature of the whole CoFeB layer.

\section{Acknowledgements}

We thank T. Hauet for the help of SQUID measurement. We also acknowledge Prof. Mair Chshiev for helpful discussion on $\mathrm{MgO}$ spin filtering effect. This work was supported by the France-China ANR-NSFC research project SISTER (Grant No. ANR-11-IS10-0001), and French ANR research project INSPIRE (Grant No. ANR-10-BLAN1014). X. F. Han also acknowledges the support of Chinese State Key Project of Fundamental Research of Ministry of Science and Technology (MOST, No. 2010CB934401). 


\section{References:}

${ }^{1}$ S. Datta, B. Das, Applied Physics Letters 56,665 (1990).

${ }^{2}$ R. Fiederling, M. Keim, G. Reuscher, W. Ossau, G. Schmidt, A. Waag, L.W. Molenkamp, Nature 402, 787 (1999).

${ }^{3}$ A. T. Hanbicki, O. M. J. van ’t Erve, R. Magno, G. Kioseoglou, C. H. Li, B. T. Jonker, G. Itskos, R. Mallory, M. Yasar, A. Petrou, Appl. Phys. Lett. 82, 4092 (2003).

${ }^{4}$ O. M. J. van 't Erve, G. Kioseoglou, A. T. Hanbicki, C. H. Li, B. T. Jonker, R. Mallory, M. Yasar, A. Petrou, Appl. Phys. Lett. 84, 4334 (2004).

${ }^{5}$ C. Adelmann, X. Lou, J. Strand, C. J. Palmstrøm, P. A. Crowell, Phys. Rev. B 71, 121301R (2005).

${ }^{6}$ X. Jiang, R. Wang, R. M. Shelby, R. M. Macfarlane, S. R. Bank, J. S. Harris, S. S. P. Parkin, Phys. Rev. Lett. 94, $056601(2005)$.

${ }^{7}$ L. Lombez, P. Renucci, P. F. Braun, H. Carrère, X. Marie, T. Amand, B. Urbaszek, J. L. Gauffier, P. Gallo, T. Camps, A. Arnoult, C. Fontaine, C. Deranlot, R. Mattana, H. Jaffrès, J.-M. George, P. H. Binh, Appl. Phys. Lett. 90, 081111 (2007).

${ }^{8}$ Y. Lu, V. G. Truong, P. Renucci, M. Tran, H. Jaffrès, C. Deranlot, J.-M. George, A. Lemaître, Y. Zheng, D. Demaille, P.-H. Binh, T. Amand, X. Marie, Appl. Phys. Lett. 93, 152102 (2008).

${ }^{9}$ V.G. Truong, PH. Binh, P. Renucci, M. Tran, H. Jaffrès, Y. Lu, C. Deranlot, J.-M. George, A. Lemaître, T. Amand, X. Marie, Appl. Phys. Lett. 94, 141109 (2009).

${ }^{10}$ E. D. Fraser, S. Hegde, L. Schweidenback, A. H. Russ, A. Petrou, H. Luo, G. Kioseoglou, Appl. Phys. Lett. 97, $041103(2010)$.

${ }^{11}$ N. Nishizawa, K. Nishibayashi and H. Munekata, Appl. Phys. Lett. 104, 111102 (2014).

${ }^{12}$ N. Nishizawa and H. Munekata, J. Appl. Phys. 114, 033507 (2013).

${ }^{13}$ M. Holub, J. Shin, D. Saha, P. Bhattacharya, Phys. Rev. Lett 98, 146603 (2007).

${ }^{14}$ A. Fert, H. Jaffrès, Phys. Rev. B 64, 184420 (2001).

${ }^{15}$ W. H. Butler, X.-G. Zhang, T. C. Schulthess, and J. M. MacLaren, Phys.Rev. B 63, 054416 (2001).

${ }^{16}$ S. Yuasa, T. Nagahama, A. Fukushima, Y. Suzuki, and K. Ando, Nat.Mater.3, 868 (2004).

${ }^{17}$ S. S. P. Parkin, C. Kaiser, A. Panchula, P. M. Rice, B. Hughes, M. Samant,and S.-H. Yang, Nat. Mater. 3, 862 2004.

${ }^{18}$ Y. S. Choi, K. Tsunekawa, Y. Nagamine, and D. Djayaprawira, J. Appl. Phys. 101, 013907 (2007).

${ }^{19}$ S. Ikeda, J. Hayakawa, Y. Ashizawa, Y.M. Lee, K. Miura, H. Hasegawa, M. Tsunoda, F. Matsukura and H. Ohno, Appl. Phys. Lett. 93, 082508 (2008). 
${ }^{20}$ D. D. Djayaprawira, K. Tsunekawa, M. Nagai, H. Maehara, S. Yamagata,N. Watanabe, S. Yuasa, Y. Suzuki, and K. Ando, Appl. Phys. Lett. 86,092502 (2005).

${ }^{21}$ S. Yuasa, Y. Suzuki, T. Katayama, and K. Ando, Appl. Phys. Lett. 87, 242503 (2005).

${ }^{22}$ W. G. Wang, S. Hageman, M. Li, S. X. Huang, X. M. Kou, X. Fan, John Q. Xiao, C. L. Chien, Appl. Phys. Lett. 99, 102502. (2011).

${ }^{23}$ W. R. Mason, A practical guide to magnetic circular dichroism spectroscopy, Wiley-Interscience, 2007.

${ }^{24}$ M. I. Dyakonov, Spin physics in semiconductors, Springer, Berlin, Germany 2008.

${ }^{25}$ R. Wang, X. Jiang, R. M. Shelby, R. M. Shelby, R. M. Macfarlane, and S. S. P. Parkin, S. R. Bank and J. S. Harris, Appl. Phys. Lett. 86, 052901 (2005).

${ }^{26}$ B. L. Liu, P. Renucci, H. Carrere, M. Senes, X. Marie, J. F. Bobo, C. Fontaine, A. Arnoult, P. H. Binh, T. Amand, Physica Status Solidi C 3, 475 (2004).

${ }^{27}$ G. Salis, R. Wang, X. Jiang, R. M. Shelby, S. S. P. Parkin, S. R. Bank, and J. S. Harris, Appl. Phys. Lett. 87, $262503(2005)$

${ }^{28}$ C. H. Li, G. Kioseoglou, A. Petrou, M. Korkusinski, P. Hawrylak and B. T. Jonker., Appl. Phys. Lett 103, 212403 (2013)

${ }^{29}$ T. Miyajima, T. Ibusuki, S. Umehara, M. Sato, S. Eguchi, M. Tsukada, and Y. Kataoka, Appl. Phys. Lett. 94, 122501 (2009).

${ }^{30}$ M. Yamanouchi, A. Jander, P. Dhagat, S. Ikeda, F. Matsukura, H. Ohno, IEEE Magn. Lett. 2, 3000304 (2011).

${ }^{31}$ Y. Jang, C. Nam, K.-S. Lee, B. K. Cho, Y. J. Cho, K.-S. Kim, and K. W. Kim, Appl. Phys. Lett. 91, 102104 (2007).

${ }^{32}$ H. Meng, W. H. Lum, R. Sbiaa, S. Y. H. Lua, H. K. Tan, J. Appl. Phys. 2011, 110, 033904.

${ }^{33}$ Y. Yang, W. X. Wang, Y. Yao, H. F. Liu, H. Naganuma, T. S. Sakul, X. F. Han, and R. C. Yu, Appl. Phys. Lett. $101,012406(2012)$. 
Figure captions

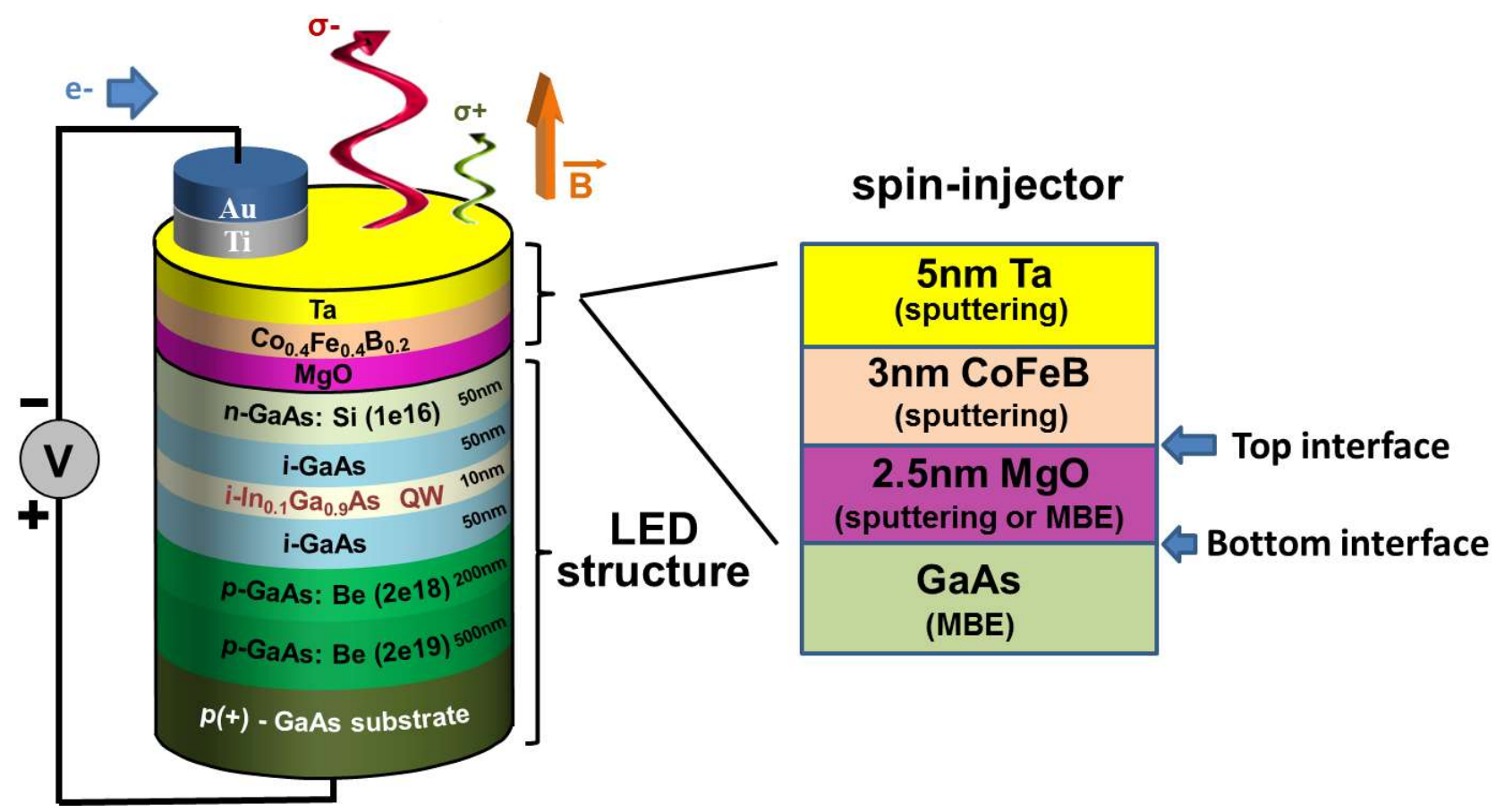

FIG. 1. (Color online). Spin-LED structure with a single InGaAs/GaAs QW. The $\mathrm{CoFeB} / \mathrm{MgO} / \mathrm{GaAs}$ spin injector layers are decomposed in terms of a top interface $\mathrm{CoFeB} / \mathrm{MgO}$ and a bottom interface $\mathrm{MgO} / \mathrm{GaAs}$. 


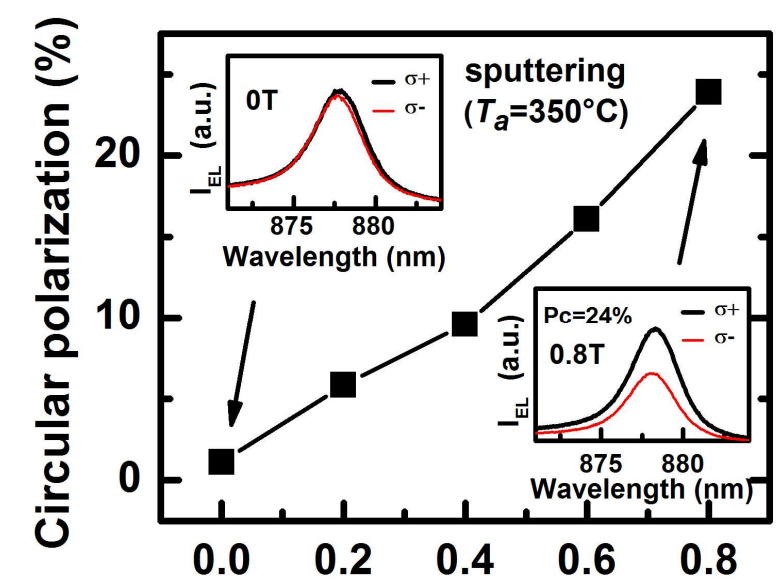

(a) Magnetic field (T)

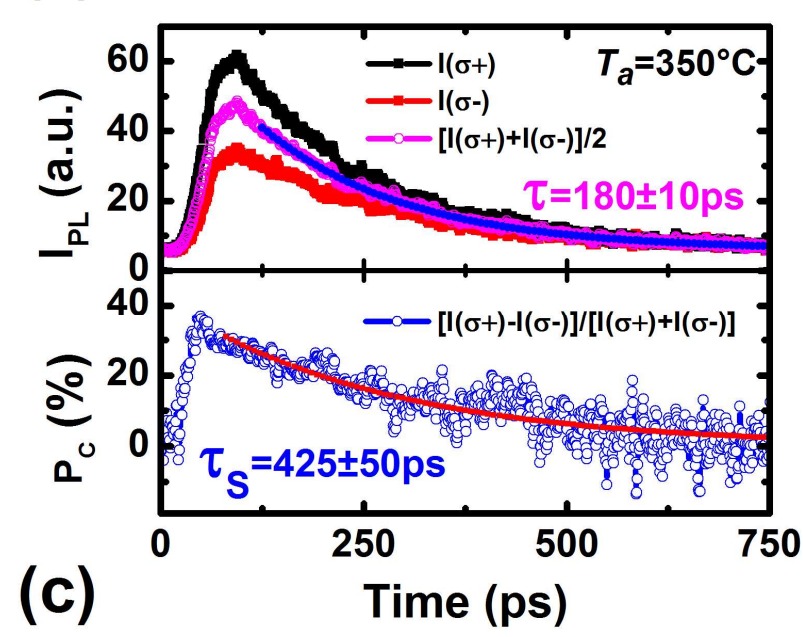

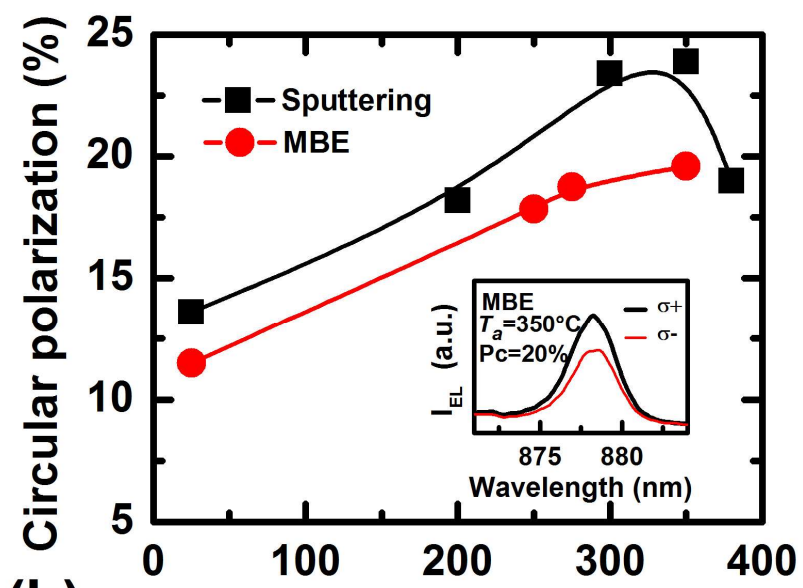

(b) Annealing temperature $\left({ }^{\circ} \mathrm{C}\right)$

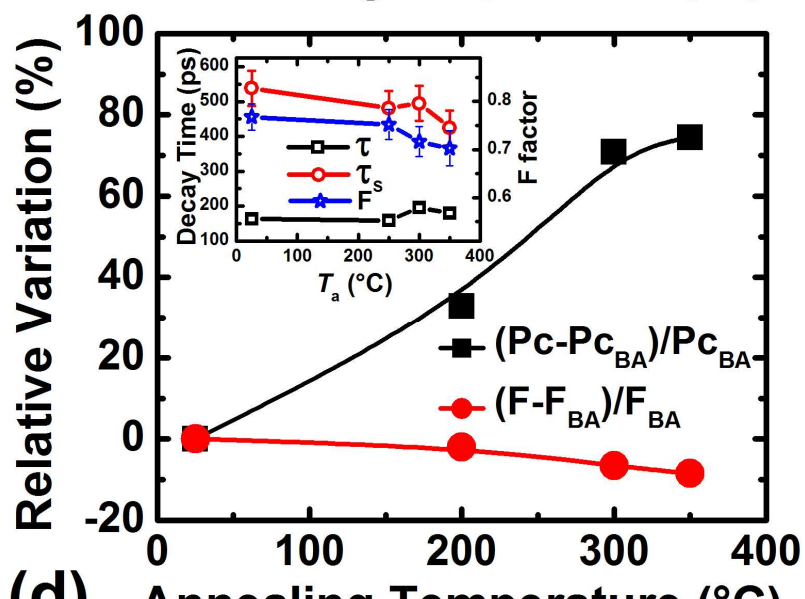

FIG. 2. (Color online) (a) EL circular polarization $P_{\mathrm{c}}$ as a function of the applied longitudinal magnetic field for the spin-LED with a sputtered $\mathrm{MgO}$ tunnel barrier $\left(T_{\mathrm{a}}=350^{\circ} \mathrm{C}\right)$ at $T=25 \mathrm{~K}$. EL spectra at zero magnetic field (top inset) and at $B=0.8 \mathrm{~T}$ (bottom inset) for $I^{\sigma+}$ (thick black line) and $I^{\sigma-}$ (thin red line) EL components. (b) EL circular polarization $P_{\mathrm{c}}$ as a function of the annealing temperature for sputtering (black squares) and MBE (red circles) grown $\mathrm{MgO}$ spin-LEDs at $T=25 \mathrm{~K}$. Inset: EL spectra of a spin-LED with a $\mathrm{MBE}$ grown tunnel barrier $\left(T_{\mathrm{a}}=350^{\circ} \mathrm{C}\right)$ at $T=25 \mathrm{~K}$ and $B=0.8 \mathrm{~T}$ for $I^{\sigma^{+}}$(thick black line) and $I^{\sigma-}$ (thin red line) EL components. (c) TRPL measurement on a bare $p$-i-n LED sample $\left(T_{\mathrm{a}}=350^{\circ} \mathrm{C}\right)$ at $T=25 \mathrm{~K}$. Top: PL intensity components $I^{\sigma^{+}}$and $I^{\sigma-}$ with respectively $\sigma^{+}$ polarization (black squares) and $\sigma^{-}$polarization (red squares) as a function of time after a $1.5 \mathrm{ps}$ laser $\left(\sigma^{+}\right)$pulsed excitation at $780 \mathrm{~nm}$ (above GaAs bandgap). The sum of the two intensities components $I_{\text {sum }}=I^{\sigma+}+I^{\sigma-}$ is displayed in pink opened circles. The decay is characterized by the electron lifetime $\tau$. Bottom: time evolution of $P_{\mathrm{c}}$ of PL at $T=25 \mathrm{~K}$ (blue opened circles). The decay is characterized by the electron spin relaxation time $\tau_{\mathrm{s}}$. (d) Relative variation of the EL $P_{\mathrm{c}}$ (black squares) for sputtered samples and of the $F$ factor (red circles) as a function of $T_{\mathrm{a}}$. Inset: Electron spin relaxation time $\tau_{\mathrm{s}}$ (red open circles), electron lifetime $\tau$ (black open squares) and $F$ factor (blue open stars) as a function of the annealing temperature $T_{\mathrm{a}}$. 


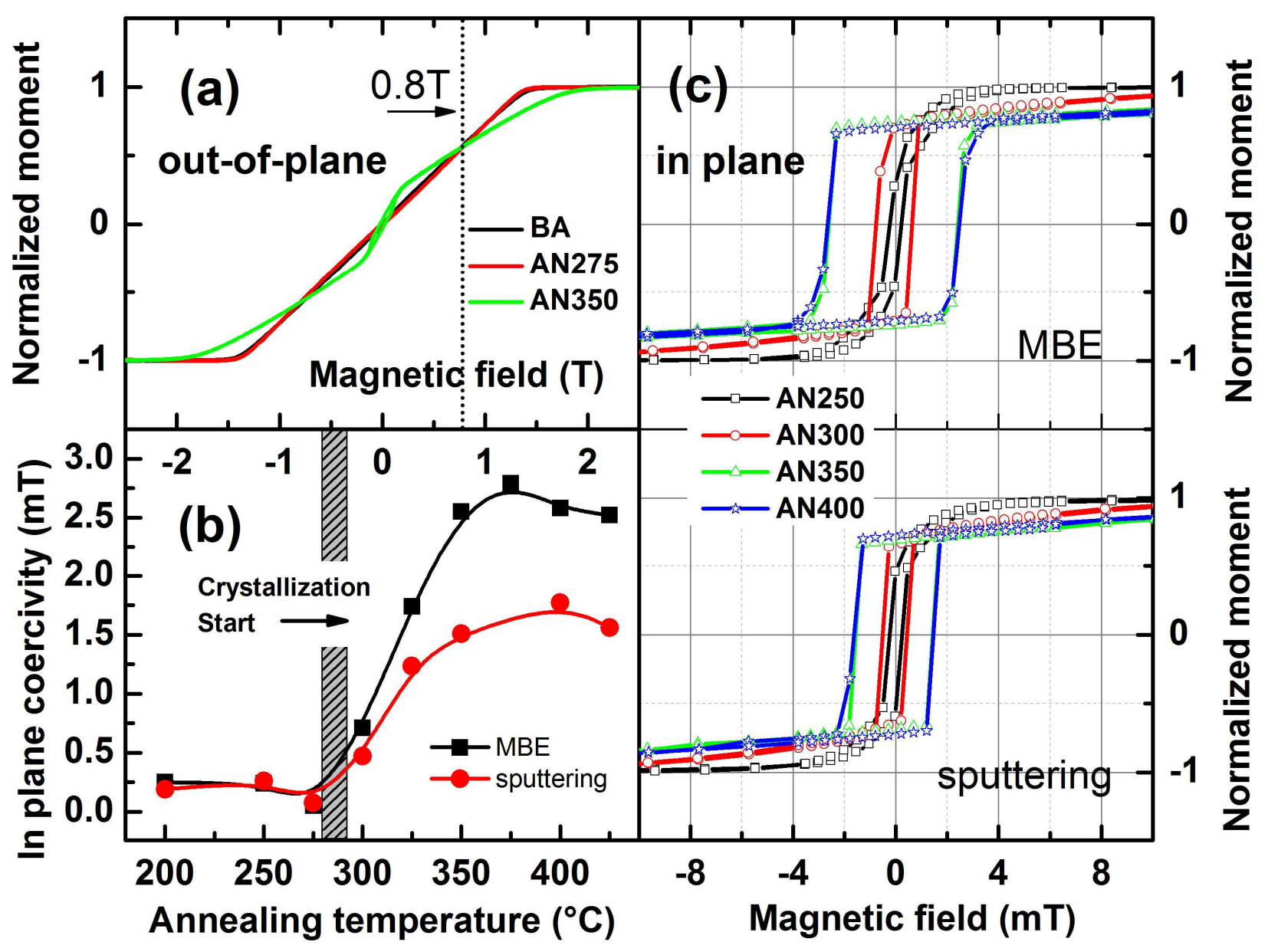

FIG. 3. (Color online) SQUID measurement at $T=300 \mathrm{~K}$. (a) Normalized magnetization as a function of the applied out-of-plane magnetic field for a sputtering spin-LED before annealing (black line) and annealed at $275^{\circ} \mathrm{C}$ (red line) and $350^{\circ} \mathrm{C}$ (green line). (b) In-plane coercivity field $H_{\mathrm{c}}$ as a function of the annealing temperature $T_{\mathrm{a}}$ for a spin-LED with sputtered $\mathrm{MgO}$ (red circles) and with $\mathrm{MBE}$ grown $\mathrm{MgO}$ (black squares). (c) Top: In-plane hysteresis loop of spin-LED with MBE grown MgO annealed at different temperatures. Bottom: Same measurements for a spin-LED with sputtered $\mathrm{MgO}$ tunnel barrier. 



FIG. 4. (Color online) HR-TEM images of (a) sample with MBE grown MgO and (c) sample with sputtered MgO before annealing. Insets: RHEED patterns on $\mathrm{MgO}$ surface from GaAs [100] and [110] azimuths (left and right insets respectively). (b) HR-TEM image of the sample with MBE grown $\mathrm{MgO}$ after annealing at $T_{\mathrm{a}}=350^{\circ} \mathrm{C}$. Left inset: FFT pattern on the square zone in CoFeB layer. Right inset: HR-STEM image to show the crystallographic orientation relationship between GaAs, $\mathrm{MgO}$ and CoFe. (d) HR-TEM image of the sample with sputtered $\mathrm{MgO}$ after annealing at $T_{\mathrm{a}}=350^{\circ} \mathrm{C}$. Left inset: FFT pattern on the square zone in CoFeB layer. Right inset: TEM image with a large scale to show the homogeneity of structures. 